measures. The study illustrates that in times of crisis, such as the COVID19 pandemic, the burden of effective infection control requires much more multidisciplinary efforts to prevent unintentional lapses in patient safety. A swift response by the state and local health departments at a tertiary-care healthcare center conveyed a positive mitigation of the highest clinical threats and decreased horizontal transmission of disease.

Funding: No

\section{Disclosures: None}

Antimicrobial Stewardship \& Healthcare Epidemiology 2021;1(Suppl. S1): doi:10.1017/ash.2021.17

\section{Presentation Type:}

Poster Presentation - Top Poster Award

Subject Category: COVID-19

A Cluster of Aspergillosis Associated with SARS-CoV-2

Kerrie VerLee; Jim Codman; Russell Lampen; Chau Nguyen; Tunisia Peters; Greg Kruse; Derek VanderHorst; Doreen Marcinek and Molly Kane-Carbone

Background: Coronavirus disease 2019 (COVID-19) has demonstrated a variety of presentations and clinical complications, among them coinfection of pneumonia with the mold Aspergillus spp. Patients at risk for invasive disease include transplant recipients and those with prolonged neutropenia, immune disorders, cystic fibrosis, and steroid use. There have been recent descriptions of coronavirus disease-associated pulmonary aspergillosis (CAPA). An outbreak investigation into a cluster of Aspergillus fumigatus infections in a health system intensive care unit uncovered a community-onset (CO) increase in CAPA. Methods: A multidisciplinary outbreak investigation was conducted evaluating sources of contamination, completion of construction projects, and changes in clinical processes. Retrospective chart review was done for the prior 18 months and incidence density rates for Aspergillus infections from June 2019 through December 2020 were calculated per 10,000 patient days, stratified by unit, specimen source, and coinfection with COVID-19. Data were linked with all positive and negative COVID-19 tests performed by the health system's regional laboratory from March to December 2020. Healthcare-onset (HO) classification was based on infections identified $\geq 7$ days after admission. Statistical analysis was calculated with significance at $\mathrm{p}<0.05$. Results: Over the last 18 months, 82 patients were identified positive with Aspergillus cultures; of which 10 (12\%) met CAPA definitions. Aspergillus fumigatus was the most common species and accounted for $62 \%$ of samples, followed by Aspergillus niger (17\%). Median rates of HO Aspergillosis were 0.45 cases per 10,000 patient days, whereas the median total rates of infection were 1.97 cases per 10,000 patient days. Rates of CAPA coincided with COVID-19 hospitalization rates. In the spring and fall, surges of COVID-19, the rate ratio of CAPA to COVID hospitalized infections per 10,000 patient days, ranged from 0.006 to 0.015. Once CAPA infections were adjusted for, rates of CO Aspergillus remained high, whereas $\mathrm{HO}$ cases suggested baseline acquisition. Conclusion: This study outlines rates of $\mathrm{CO}$ aspergillosis as well as CAPA rates coinciding with the healthcare system's spring and fall surges of COVID-19 hospitalizations. Despite the determination that this was not a hospital-acquired cluster, the investigation revealed some areas for

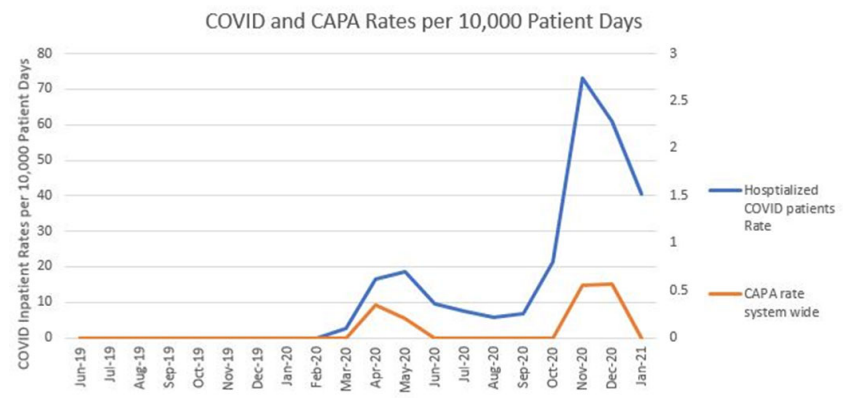

Figure 1.

opportunity in construction processes along with maintaining coverage of all patient supplies to reduce the risk of contamination.

Funding: No

Disclosures: None

Antimicrobial Stewardship \& Healthcare Epidemiology 2021;1(Suppl. S1):s10

doi:10.1017/ash.2021.18

\section{Presentation Type:}

Poster Presentation - Top Poster Award

Subject Category: COVID-19

Molecular Epidemiology of Large COVID-19 Clusters at an Academic Medical Center, March-October 2020

Takaaki Kobayashi; Miguel Ortiz; Stephanie Holley; William Etienne; Kyle Jenn; Oluchi Abosi; Holly Meacham; Lorinda Sheeler; Angie Dains; Mary Kukla; Alexandra Trannel; Alexandre Marra; Mohammed Alsuhaibani; Paul McCray; Stanley Perlman; Bradley Ford; Daniel Diekema; Melanie Wellington; Alejandro Pezzulo and Jorge Salinas

Background: COVID-19 in hospitalized patients may be the result of community acquisition or in-hospital transmission. Molecular epidemiology can help confirm hospital COVID-19 transmission and outbreaks. We describe large COVID-19 clusters identified in our hospital and apply molecular epidemiology to confirm outbreaks. Methods: The University of Iowa Hospitals and Clinics is an 811-bed academic medical center. We identified large clusters involving patients with hospital onset COVID-19 detected during March-October 2020. Large clusters included $\geq 10$ individuals (patients, visitors, or HCWs) with a laboratory confirmed COVID-19 diagnosis (RT-PCR) and an epidemiologic link. Epidemiologic links were defined as hospitalization, work, or visiting in the same unit during the incubation or infectious period for the index case. Hospital onset was defined as a COVID-19 diagnosis $\geq 14$ days from admission date. Admission screening has been conducted since May 2020 and serial testing (every 5 days) since July 2020. Nasopharyngeal swab specimens were retrieved for viral whole-genome sequencing (WGS). Cluster patients with a pairwise difference in $\leq 5$ mutations were considered part of an outbreak. WGS was performed using Oxford Nanopore Technology and protocols from the ARTIC network. Results: We identified 2 large clusters involving patients with hospital-onset COVID-19. Cluster 1: 2 hospital-onset cases were identified in a medical-surgical unit in June 2020. Source and contact tracing revealed 4 additional patients, 1 visitor, and 13 employees with COVID-19. Median age for patients was 62 (range, $38-79$ ), and all were male. In total, 17 samples (6 patients, 1 visitor, and 10 HCWs) were available for WGS. Cluster 2: A hospital-onset case was identified via serial testing in a non-COVID-19 intensive care unit in September

\section{number of mutations}

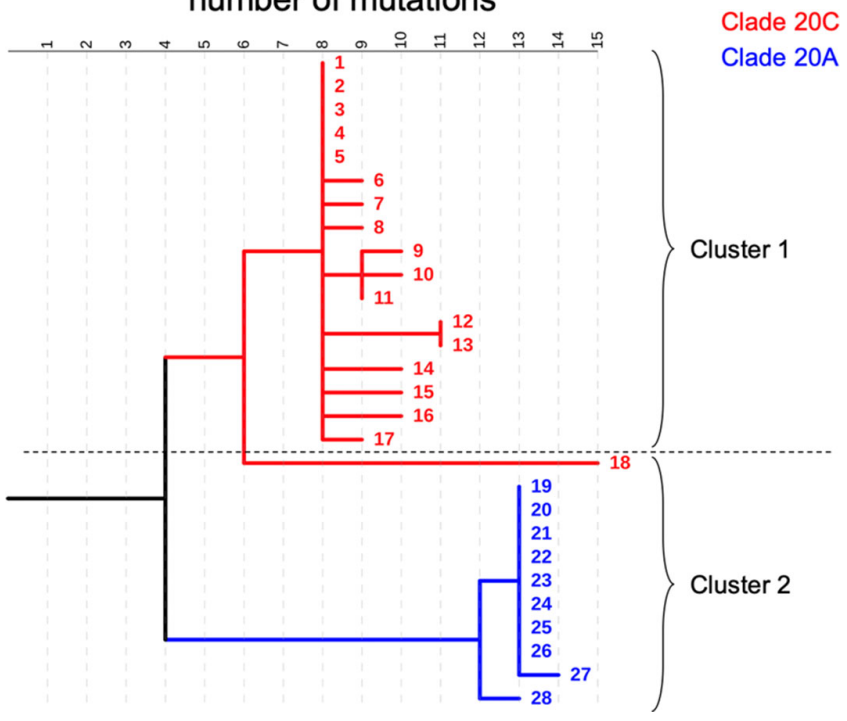

Figure 1.

(c) The Author(s), 2021. Published by Cambridge University Press on behalf of The Society for Healthcare Epidemiology of America. This is an Open Access article, distributed under the terms of the Creative Commons Attribution licence (http://creativecommons.org/licenses/by/4.0/), which permits unrestricted re-use, distribution, and reproduction in any medium, provided the original work is properly cited.

S10 2021;1 Suppl 1 
2020. Source investigation, contact tracing, and serial testing revealed 3 additional patients, and $8 \mathrm{HCWs}$. One HCW also had a community exposure. Patient median age was 60 years (range, 48-68) and all were male. In total, 11 samples (4 patients and 7 HCWs) were sequenced. Using WGS, cluster 1 was confirmed to be an outbreak: WGS showed 0-5 mutations in between samples. Cluster 2 was also an outbreak: WGS showed less diversity (0-3 mutations) and ruled out the HCW with a community exposure (20 mutations of difference). Conclusion: Whole-genome sequencing confirmed the outbreaks identified using classic epidemiologic methods. Serial testing allowed for early outbreak detection. Early outbreak detection and implementation of control measures may decrease outbreak size and genetic diversity. Funding: No

Disclosures: None

Antimicrobial Stewardship \& Healthcare Epidemiology 2021;1(Suppl. S1):s10-s11

doi:10.1017/ash.2021.19

Presentation Type:

Poster Presentation - Top Poster Award

Subject Category: COVID-19

COVID-19 Vaccination of HCWs in the First Phase of a Large-Scale Mass Vaccination Program within a Healthcare System

Kimberly Korwek; E. Jackie Blanchard; Julia Moody; Katherine Lange; Ryan Sledge; Maria Benedetti and Kenneth Sands

Background: The approval of the first SARS-COV-2 vaccines for COVID-19 were accompanied by unprecedented efforts to provide vaccination to healthcare workers and first responders. More information about vaccine uptake in this group is needed to better refine and target educational messaging. Methods: HCA Healthcare used federal guidance and internal experience to create a systemwide mass vaccination strategy. A closed point-of-dispensing (POD) model was developed and implemented. The previously developed enterprise-wide emergency operations strategy was adapted and implemented, which allowed for rapid development of communications and operational processes. A tiering strategy based on recommendations from the National Academies was used in conjunction with human resources data to determine vaccine eligibility for the first phase of vaccination. A comprehensive data and reporting strategy was built to connect human resources and vaccine consent data for tracking vaccination rates across the system. Results: Vaccination of employed and affiliated colleagues began December 15, 2020, and was made available based on state-level release of tiers. Within the first 6 weeks, in total, 203,544 individuals were eligible for vaccine based on these criteria. Of these, $181,282(89.1 \%)$ consented to and received vaccine, 19,788 (9.7\%) declined, and 2,474 (1.2\%) indicated that they had already been vaccinated. Of those eligible, the highest acceptance of vaccine was among the job codes of specialists and professionals ( $\mathrm{n}=7,914$ total, $100 \%$ consent), providers $(\mathrm{n}=23,335,99.6 \%$ ), and physicians $(\mathrm{n}=3,218,98.4 \%)$. Vaccine was most likely to be declined among job codes of clerical and other administrative ( $\mathrm{n}=12,889$ total, $80.1 \%$ consent), clinical specialists and professionals $(\mathrm{n}=$ $22,853,81.0 \%$, ) and aides, orderlies and technicians ( $\mathrm{n}=17,803,82.6 \%$,). Registered nurses made up the largest eligible population $(\mathrm{n}=56,793)$, and $89.5 \%$ of those eligible consented to receive vaccination. Average age among those who consented was slightly older ( 48.3 years) than those that declined (44.7 years), as was length of employment tenure (6.9 vs 5.0 years). Conclusion: A large-scale, closed POD, mass vaccination program was able to vaccinate nearly 200,000 healthcare workers for SARS-CoV-2 in 6 weeks. This program was implemented in acute-care sites across 20 different US states, and it was able to meet the various state-level requirements for management of processes, product, and required reporting. The development of a standardized strategy and custom, centralized monitoring and reporting facilitated insight into the characteristics of early vaccine adopters versus those who decline vaccination. These data can aid in the refining and targeting of educational materials and messaging about the SARS-CoV-2 vaccine.

Funding: No

Disclosures: None

Antimicrobial Stewardship \& Healthcare Epidemiology 2021;1(Suppl. S1):s11

doi:10.1017/ash.2021.20
Presentation Type:

Poster Presentation - Top Poster Award

Subject Category: Disinfection/Sterilization

High-Level Disinfection in Ambulatory Care: Overcoming the Barriers of a Decentralized System through Auditing and Education

Sonja Rivera Saenz

Background: High-level disinfection (HLD) of semicritical instruments in a multispecialty ambulatory care network has the potential for increased risk due to the decentralized instrument reprocessing and lack of a sterile processing department. Attention to HLD practices is an important part of device-borne outbreak prevention. Method: An HLD database was developed to identify specific departments and locations where HLD occurred across a 30-medical practice ambulatory care network in eastern Massachusetts, which included otolaryngology, urology, endoscopy, and obstetrics/gynecology departments. Based on qualitative feedback from managers and reprocessing staff, this database centralized information that included the supply inventory including manufacturer and model information, HLD methodology, standard work, and listing of competency evaluations. The infection control team then led audits to directly observe compliance with instrument reprocessing and a monthly-driven HLD calendar was developed to enforce annual competencies. Result: The results of the audits demonstrated variability across departments with gaps in precleaning, transportation of used instruments, the dilution of enzymatic cleaner, and maintenance of quality control logs. Given the uniqueness of shape and size of various ambulatory locations, proper storage and separation between clean and dirty spaces were common pitfalls. Auditing also revealed different levels of staff understanding of standard work and variable inventory management. Centralized education sessions held jointly by the infection control team and various manufacturers for the reprocessing staff helped to create and reinforce best practices. Conclusion: Decentralized HLD that occurs across multiple ambulatory care sites led to gaps in instrument reprocessing and unique challenges due to variable geography of sites, physical space constraints, and an independent approach to procuring medical supplies. Through the auditing and feedback of all areas that perform HLD, an effective and sustainable strategy was created to ensure practice improvement. Streamlining standard work, seeking direct input from frontline staff, and collective educational events were critical to our success in the ambulatory setting.

Funding: No

Disclosures: None

Antimicrobial Stewardship \& Healthcare Epidemiology 2021;1(Suppl. S1):s11

doi:10.1017/ash.2021.21

\section{Presentation Type:}

Poster Presentation - Top Poster Award

Subject Category: Long-Term Care

Coronavirus in Wisconsin Nursing Homes: A Longitudinal Analysis of the First 10 Months of the Pandemic

Cameron Gmehlin; Frida Rivera; Jorge Ramos-Castaneda; Liliana Pezzin; EDmund Duthie and L. Silvia Munoz-Price

Background: The COVID-19 pandemic has disproportionately affected nursing home residents, and emerging evidence suggests quality, location, resident demographics, and staffing levels may be related to COVID-19 incidence within facilities. We describe the distribution of COVID-19 cases in Wisconsin nursing homes from January 2020 to October 2020, the effect of rural versus urban locations on COVID-19 incidence, and the temporal changes in COVID-19 incidence. Methods: We constructed a database using the Center for Medicaid and Medicare Services' (CMS) publicly available data. Variables obtained per facility included location, number of beds, ownership type, average census, 5-star ratings (overall, quality, health, staffing, and nurse staffing categories), number of COVID-19 cases, resident Medicaid/Medicare share, area deprivation index, and social vulnerability index. Nursing homes were divided into tertiles based on total COVID-19 cases for descriptive analysis (zero cases, $1-7$ cases, $>7$ cases). Demographic and clinical variables were reported as frequencies, mean 